\title{
CORRELAÇÃO ENTRE DISFUNÇÃO TEMPOROMANDIBULAR, POSTURA E QUALIDADE DE VIDA
}

\author{
CORRELATION BETWEEN TEMPOROMANDIBULAR DYSFUNCTION, \\ CERVICAL POSTURE AND QUALITY OF LIFE
}

Daniela Aparecida Biasotto-Gonzalez*

Daniel Ventura de Andrade*

Tabajara de Oliveira Gonzalez**

Manoela Domingues Martins*

Kristianne Porta Santos Fernandes*

João Carlos Ferrari Corrêa*

Sandra Kalil Bussadori*

\begin{abstract}
Biasotto-Gonzalez DA, Andrade DVde, Gonzalez TdeO, Martins MD, Fernandes KPS, Corrêa JCF, Bussadori SK. Correlação entre disfunção temporomandibular, postura e qualidade de vida. Rev Bras Crescimento Desenvol Hum. 2008; 18(1): 79-86.
\end{abstract}

Resumo: Introdução: As disfunções temporomandibulares apresentam manifestações clínicas variadas, porém com alta prevalência de alterações posturais e impacto na qualidade de vida destes indivíduos. Objetivo: Caracterizar o grau de disfunção temporomandibular (DTM) e relacionar com a postura cervical e com a qualidade de vida em universitários. Método: Foram selecionados 98 indivíduos, de ambos os gêneros, com faixa etária entre 18 a 33 anos, através dos questionários, Índice Anamnético de Fonseca, que gradua a severidade da DTM e a versão brasileira do questionário de qualidade de Vida SF-36. A análise da postura de cabeça e pescoço foi realizada pelo software Alcimagem ${ }^{\grave{ }}$, que oferece uma análise quantitativa dos ângulos. Resultados: 68,36\% apresentaram DTM Leve, 23,47\% Moderada e 8,16\% Severa;os mesmos apresentaram prejuízo principalmente nos domínios Vitalidade e Dor $\left(X^{2}=0,03\right)$, sendo que Aspectos Emocionais e Sociais foram os domínios mais preservados. A média do ângulo cervical em indivíduos com DTM foi $90,76^{\circ}( \pm 7,72)$, sendo que há um aumento do ângulo cervical relacionado ao aumento do grau de severidade da DTM, porém não significante estatisticamente $(r=0,09)$. Conclusão: Houve uma prevalência maior de Disfunção Temporomandibular (DTM) leve, sendo que em relação ao ângulo cervical, houve um aumento com a severidade do grau de DTM, com piora da qualidade de vida nesta população.

Palavras-chave: Síndrome da disfunção da articulação temporomandibular; qualidade de vida; postura.

\section{INTRODUÇÃO}

AArticulação Temporomandibular (ATM) é um elemento do sistema estomatognático for- mado por várias estruturas internas e externas, capaz de realizar movimentos complexos. A mastigação, a deglutição, a fonação e a postura, dependem muito da função, saúde e estabilidade

\footnotetext{
* Programa de Mestrado em Ciências da Reabilitação da Universidade Nove de Julho - UNINOVE. São Paulo, SP E-mail: dani_atm@uninove.br - End: Av. Francisco Matarazzo, 612, Água Branca, São Paulo, SP, CEP. 05001-100.

** Curso de fisioterapia da Universidade de Mogi das Cruzes - UMC

Trabalho realizado no Depto. de Ciências da Reabilitação da Universidade Nove de Julho - UNINOVE - São Paulo, SP. Apoio financeiro: Cnpq e Fapic/Uninove.
} 
desta para funcionarem de forma adequada.

As desordens que acometem a articulação temporomandibular (ATM abrangem vários problemas clínicos que envolvem a musculatura da mastigação, a articulação temporomandibular (ATM) e estruturas associadas, cujas características são: dor crônica, fadiga, sensibilidade nos músculos da mastigação, ruídos e limitação de movimento, sendo responsáveis por uma sintomatologia diversificada, de difícil diagnóstico e tratamento, que envolvem manifestações de dor e incoordenação muscular, relacionada ao desequilíbrio biomecânico não apenas da própria articulação, como também de áreas circunvizinhas, incluindo-se a região cervical.

Dentre essas desordens, os dismorfismos craniofaciais apresentam entre seus tipos diferentes classes oclusais onde a posição da mandíbula tem relação direta com a postura da cabeça e ombros, por esta ser um osso livre'.

Okeson $^{2}$ sugere que anormalidades oclusais são as possíveis causas das cefaléias, DTM e dores faciais. Porém, a influência da coluna cervical nas estruturas da mastigação é freqüentemente ignorada ${ }^{3}$.

Severas desordens crânio-cervicais como anteriorização da cabeça, retificação da coluna cervical, e assimetria de ombros têm sido estabelecidas em pacientes com Disfunção Temporomandibular ${ }^{4}$.

Independentemente do tipo de avaliação clínica ou questionário aplicado nos diversos trabalhos da literatura ${ }^{1,3-5}$ pode-se evidenciar vários sinais e sintomas clínicos presentes na DTM, tais como: cefaléia, mialgias, sensibilidade à palpação dos músculos mastigatórios e das articulações temporomandibulares, zumbido, ruídos articulares durante os movimentos condilares e movimentos mandibulares limitados ou assimétricos. Esta variedade de sintomatologia clínica mostra que não há um único fator etiológico responsável pela DTM sendo, portanto, sua patogenia multifatorial, abrangendo importantes elementos funcionais, anatômicos e psicossociais ${ }^{1,3,6,7}$.

As alterações causadas pela DTM, em especial a dor, podem interferir nas atividades diárias sociais do indivíduo afetado levando a um efeito negativo na função social, na saúde emo- cional, e no nível de energia ${ }^{5,8-10}$.

Embora existam razões para acreditar que as disfunções temporomandibulares e outras condições dolorosas da face causem algum impacto na qualidade de vida, apenas um pequeno número de estudos documenta o uso de questionários específicos ou mesmo de ferramentas multidimensionais aplicados a estes pacientes ${ }^{11-13}$. Assim, o objetivo é caracterizar o grau de disfunção temporomandibular (DTM) e relacioná-lo com a postura cervical e com a qualidade de vida em universitários.

\section{MÉTODO}

Foi realizado um estudo prospectivo em 98 voluntários de uma amostra de universitários, de ambos os sexos, no período de março a setembro de 2006. Dos 98 voluntários, 44 são do sexo masculino e 54 do feminino. A idade variou de 18 a 33 anos.

A pesquisa foi realizada seguindo as normas que regulamentam pesquisa em seres humanos contidas nas Resoluções no 196/96 e 251/ 97 do Conselho Nacional de Saúde, com protocolo $n^{\circ} 105730$, sendo que os procedimentos foram previamente explicados a todos os participantes.

Aplicou-se dois questionários, sendo o primeiro descrito por Fonseca et al. ${ }^{1}$, sobre caracterização dos graus de disfunção temporaomandibular (DTM) e o segundo elaborado por Ciconelli e Ferraz ${ }^{15}$, SF-36, que avalia o impacto da qualidade de vida.

Setecentos questionários foram aplicados. Destes, 302 foram respondidos. Após análise dos mesmos, 160 foram selecionados para ingresso na pesquisa, sendo que apenas 98 foram utilizados para compor a amostra.

Os critérios de inclusão foram os seguintes: indivíduos que apresentaram sinais e sintomas relacionados à DTM (Fonseca et al. ${ }^{1}$ ), que assinaram o termo de consentimento livre e esclarecido e que concluíram todo o protocolo de coleta de dados. Foram excluídos os indivíduos que não apresentaram DTM, que estavam em tratamento fisioterapêutico e ou odontológico, bem como que apresentaram história de doen- 
ças sistêmicas como artrite reumatóide e diabetes, traumas e ou cirurgias na face, que não completaram o protocolo com a respectiva imagem fotográfica.

Para diagnóstico da DTM foi utilizado o índice anamnético de Fonseca et al. ${ }^{1}$, avaliação postural e o exame fotográfico. Para a avaliação postural, utilizaram-se marcadores articulares (Poliestireno expandido - Isopor ${ }^{\circledR}$ ) colocados em pontos anatômicos para mensuração das medidas em graus, fotografados por câmera digital Cyber-Shot DSC-P30 Sony ${ }^{\circledR}$. Estes dados foram incluídos e analisados no software Alcimagem ${ }^{\circledR}$, que quantifica as angulações articulares ${ }^{15}$. Para a avaliação da qualidade de vida, foi utilizada a versão brasileira do questionário SF-36.

Avaliação postural de cabeça e pescoço consistiu nas imagens realizadas com os indivíduos em pé, vista lateral, perfil direito, posicionado em uma marcação pré-estabelecida no chão. A máquina fotográfica foi fixada a um metro e meio do participante sobre um tripé com altura de $130 \mathrm{~cm}$ e zoom ajustável para a retirada da fotografia. Os participantes permaneceram com o tronco completamente despido ou biquíni no caso do sexo feminino, que foram identificados por marcadores de cor vermelha.

Após estas fotografias, as mesmas foram inseridas no computador e analisadas pelo software Alcimagem ${ }^{\circledR}$. Foi obtido o ângulo através do processo espinhoso da $7^{\text {a }}$ vértebra cervical manúbrio do esterno e ápice do mento ${ }^{16}$. Foram realizadas três medidas, sendo que se obtiveram as médias das mesmas (Figura 1).

Figura 1:Pontos analisados no software: Processo Espinhoso da $7^{\text {a }}$ Vértebra Cervical, Manúbrio do Esterno e Ápice do Mento.

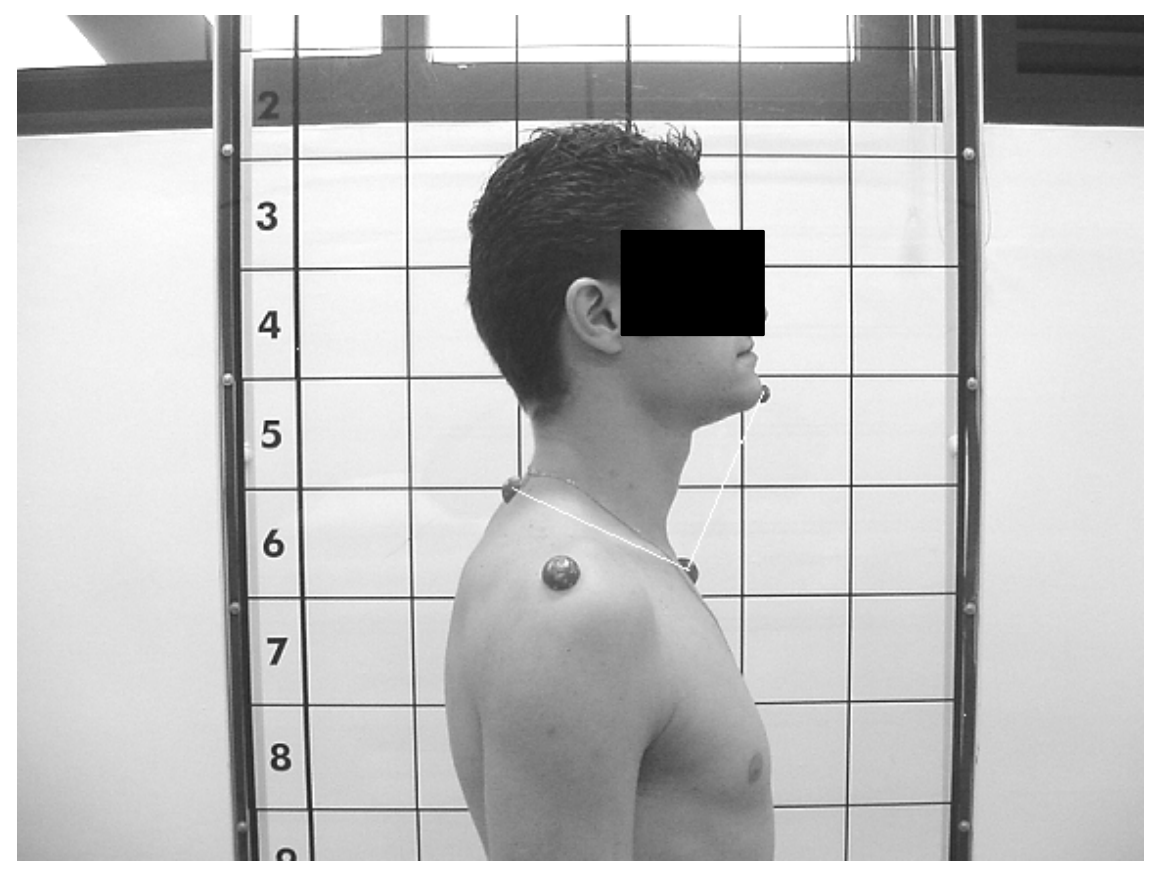

\section{Análise Estatística}

Após a aplicação do teste KolmogorovSmirnov, a amostra apresentou distribuição anormal. Foram utilizados os testes Qui-quadrado $\left(X^{2}\right)$, para avaliar a associação entre variáveis qualitativas e Correlação de Spearman para identificar a relação entre as variáveis de desfecho.

\section{RESULTADOS}

Com relação ao Índice Anamnético de Fonseca et al. ${ }^{1}$, a análise descritiva das disfunções investigadas demonstrou que $68,36 \%$ apresentaram disfunção leve, $23,47 \%$ moderada e $8,16 \%$ severa; sendo que quanto ao sexo masculino, 
$84,09 \%$ apresentam disfunção leve, $13,63 \%$ moderada e $2,27 \%$ severa; quanto ao sexo feminino, $55,55 \%$ apresentam disfunção leve, $31,48 \%$ moderada e $12,96 \%$ severa (Tabela 1).

Tabela 1: Distribuição do grau de DTM segundo o gênero.

\begin{tabular}{lll}
\hline Graus de DTM & Homem & Mulher \\
\hline Disfunção Leve & $37(84,09 \%)$ & $30(55,55 \%)$ \\
Disfunção Moderada & $6(13,63 \%)$ & $17(31,48 \%)$ \\
Disfunção Severa & $1(2,27 \%)$ & $7(12,96 \%)$ \\
Total (\%) & $44(100 \%)$ & $54(100 \%)$ \\
\hline
\end{tabular}

As análises demonstradas a seguir basearam-se na divisão por categorias, que é feita pelo questionário SF-36, apresentada previamente por Aaronson ${ }^{17}$.

As médias dos escores obtidos das categorias físicas e mentais dos sujeitos deste estudo podem ser observadas na Figura 2.

$\mathrm{O}$ aumento do ângulo cervical nos indivíduos classificados com DTM em relação ao grau de severidade pode ser observado na Figura 3.

\section{DISCUSSÃO}

Os domínios com menor valor dentre todos os componentes da qualidade de vida foram Vitalidade $(55,88)$ e Dor $(57,41)$, enquanto os domínios Aspectos Emocionais e Sociais apresentaram o maior escore $(96,71$ e 94,26$)$. Quando todos os aspectos da qualidade de vida (QV) são correlacionados com a gravidade de Disfunção Temporomandibular (DTM), há influência com maior ênfase na dor e na vitalidade. Estatisticamente há relação direta entre a presença de DTM e a QV nos indivíduos estudados $\left(X^{2}=0,03\right)$.

A Figura 3 demonstra um aumento do ângulo cervical nos indivíduos classificados com DTM em relação ao grau de severidade, porém esta correlação não é significante estatisticamente $(r=0,09$ sendo que o IC $=95 \%(-0,11$ a 0,29$))$. Estes dados ficam mais evidentes quando comparados com Gadotti et al. ${ }^{18}$, que apesar de aplicarem em indivíduos normais com diferentes classes oclusais, demonstram que a média dos ângulos cervicais é menor $36,12^{\circ}$ quando comparados com a amostra estudada $90,76^{\circ}( \pm 7,72)$.

Para avaliação e diagnóstico de Disfunção Temporomandibular na área de pesquisa epidemiológica são aplicados um grande número de escalas, questionários e índices. Dentre eles, os índices anamnéticos parecem ser ferramentas diagnósticas mais apropriadas a serem administradas nestes estudos, pois envolvem a população geral e podem fornecer informações importantes às amostras estudadas, assim como para classificar e caracterizar DTM $^{3,5}$.

Figura 2: Comparação entre os domínios do SF-36 segundo as categorias físicas (CF,AF,Dor,EGS) e mentais (V, AS,AE e SM).

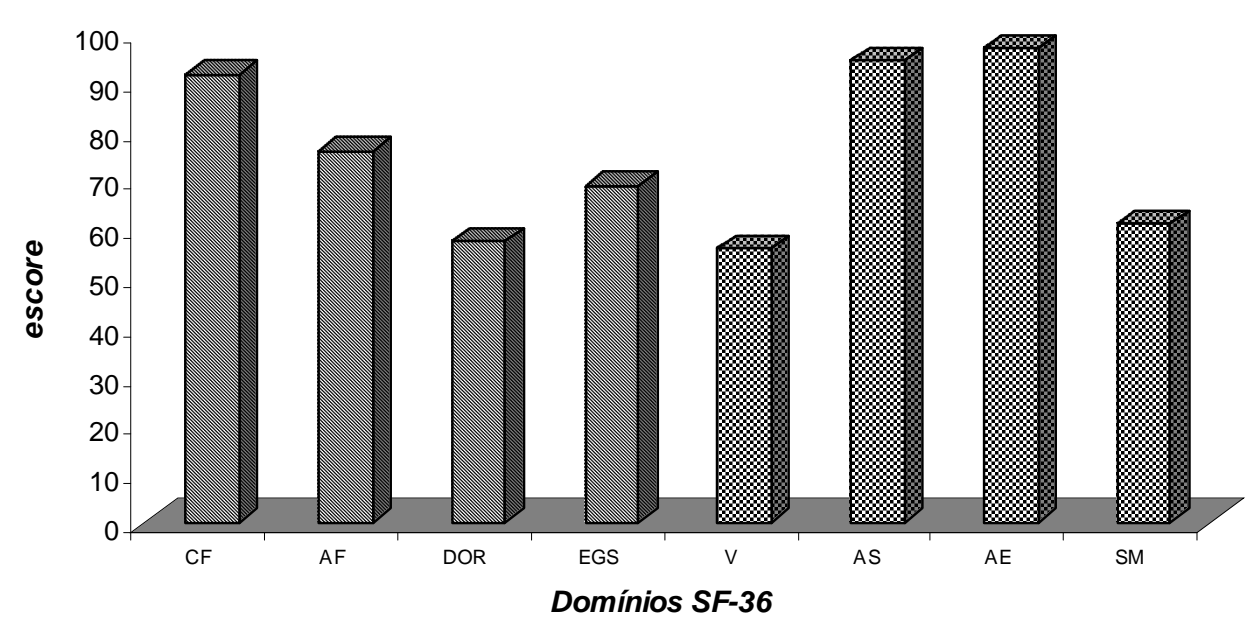


Figura 3: Ângulo cervical nos diferentes graus de DTM.

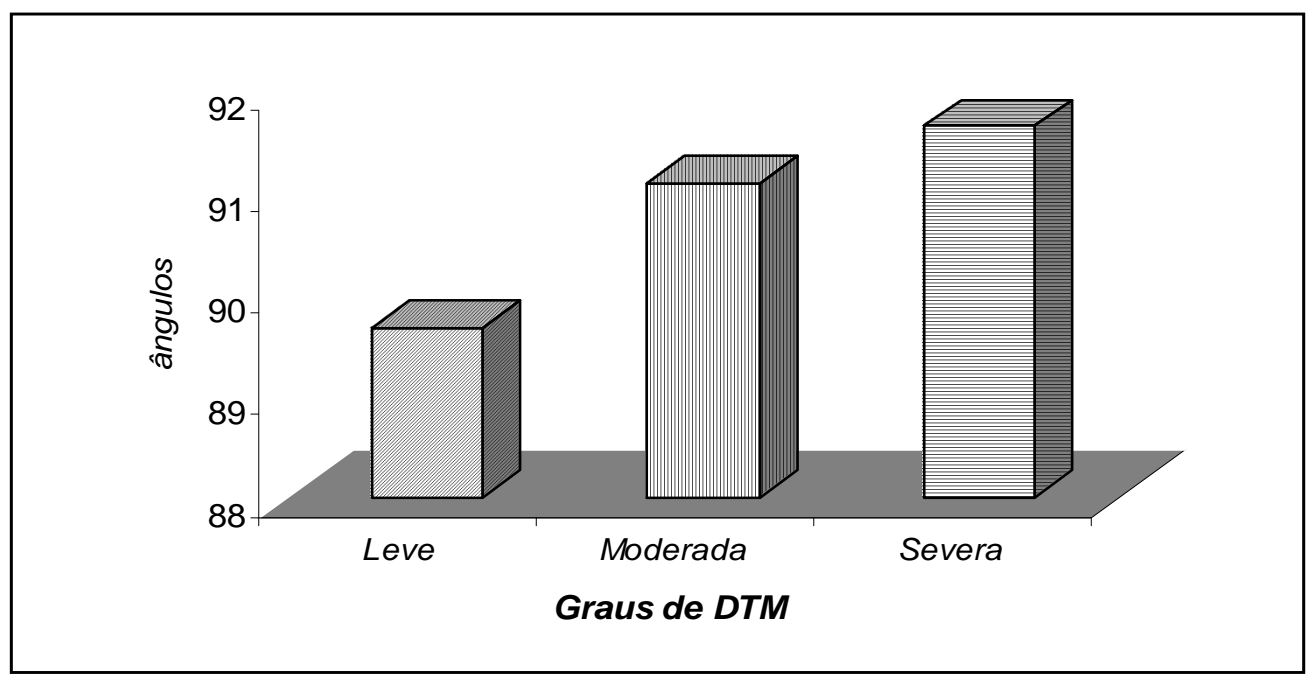

O questionário auto-administrado para avaliação de DTM desenvolvido por Fonseca et al. ${ }^{1}$, quando comparado com os critérios de diagnóstico para pesquisa das disfunções temporomandibulares, denominado RDC ou o índice de Helkimo, mostra como vantagem o menor tempo de aplicação e, portanto, um menor custo. Além disso, este índice anamnético exige menor necessidade de capacidade diagnóstica do profissional, possibilidade de uso em serviços públicos por pessoal técnico; aplicação em levantamentos epidemiológicos e de controle de tratamento ${ }^{1,19}$. Características, decisivas para adotálo nesta pesquisa.

Questionários e instrumentos numéricos têm sido utilizados para mensurar e avaliar o estado de saúde e certas limitações nas atividades das populações em geral, relatando assim suas qualidades de vida Davidson e Keating ${ }^{20}$. O SF36 é aceito internacionalmente para aplicações em pacientes e voluntários conscientes, com a mensuração sobre o estado de saúde validado ${ }^{21}$. Este questionário não demonstra o escore ideal para ser atingido por uma pessoa, mas, segundo Brazier et al. ${ }^{22}$, através dele podemos diferenciar pacientes e populações com bom ou mau estado geral de saúde.

Neste estudo observa-se que dentre as variáveis de Qualidade de Vida estudada, os aspectos Vitalidade e Dor mostram-se muito alterados, estando associados a uma pior qualidade de vida nos portadores de DTM. Estes dados também são evidentes no estudo de Oliveira et al. ${ }^{23}$, que apesar de aplicarem a versão brasileira do questionário Mc Bill de Dor (Br-MPQ) constatam que a dor da DTM tem um impacto negativo na qualidade de vida do paciente, confirmando os resultados apresentados (Figura 2). Este dado também concorda com outros estudos, os quais descrevem que o quadro de DTM associada à dor tem sido relacionado com alterações na QV ${ }^{12,} 24$.

Em relação à DTM, diversos trabalhos ${ }^{18,}$ ${ }^{25,26}$ mostram ser esta uma das principais causas de dor crônica facial e que estão intimamente relacionadas com o estresse físico e psicossomático tais como fadiga, alterações do sono, ansiedade e depressão.

Neste estudo observou-se que os valores médios totais dos domínios se encontram entre 55 e 63 pontos, com exceção dos Aspectos Emocionais e Sociais e Capacidade Funcional que, neste caso, o domínio Estado Geral da Saúde (EGS) se encontra com 68.61 pontos. Estes dados concordam com os apresentados por $\mathrm{Ku}$ roiwa et al. ${ }^{26}$ que observaram que 91 pacientes adultos com DTM e dor orofacial apresentavam escore de 54,5 pontos no questionário SF-36, em relação ao domínio EGS, ou seja, estes se encontraram em pior qualidade de vida quando comparados com os indivíduos com DTM do presente estudo. 
Quanto ao domínio Capacidade Funcional, os resultados deste trabalho mostraram um escore maior em relação ao obtido por Kuroiwa et al. ${ }^{27}$ confirmando que os aspectos Dor e Capacidade Funcional vão interferir no estado geral da saúde.

O domínio Dor (57.41) também se encontrou diminuído, porém maior do que o domínio de vitalidade (55.88). Além disso, observou-se que a dor estará influenciando de forma indireta o domínio vitalidade.

Em relação à prevalência e gravidade da disfunção temporomandibular constatou-se neste estudo que as mulheres mostram um nível de severidade maior $(12,96 \%)$ quando comparadas com os homens $(2,27 \%)$ dados esses concordantes com alguns trabalhos na literatura ${ }^{11}$, muito embora, neste estudo observou-se uma maior disfunção de DTM leve no gênero masculino (Tabela 1).

Além dos movimentos funcionais, como a mastigação e a deglutição, pôde-se encontrar também comportamentos parafuncionais representados algumas vezes pelo hábito de apertar e / ou ranger os dentes, conhecido como bruxismo ${ }^{6,7,25,28}$. Outras atitudes parafuncionais que podem influenciar na postura, e assim interferir na ATM, foi citado em um estudo realizado por Cáuas ${ }^{29}$, onde relatou que o apertamento dos dentes, morder objetos, o bruxismo, hábitos posturais como manter a cabeça em flexão em relação ao corpo, foram encontrados com maior incidência nos portadores de DTM, tendo relação com a profissão do indivíduo.

Brown ${ }^{30}$, no seu estudo, determina a variação angular da cabeça e pescoço em pacientes com e sem problemas de DTM, associada à má oclusão. Neste estudo não houve uma diferença estatisticamente significante entre os grupos, mas ocorreu uma maior variação entre o menor e o maior ângulo no grupo com DTM, diferindo do grupo controle.

As alterações posturais de cabeça e pescoço são evidentes nos indivíduos com DTM. Fato este comprovado por Biasotto-Gonzalez ${ }^{28}$ e Goldstein $^{31}$, que relatam que as influências neuromusculares das regiões cervicais e da mastigação são participativas ativamente nas funções de movimento da mandíbula e posicionamento da cervical.
Pôde-se verificar neste estudo que mesmo não sendo esta diferença estatisticamente significante, é possível observar indivíduos com DTM severa apresentando maior anteriorização de cabeça uma relação de aumento da angulação com o grau de DTM leve. Este achado é concordante com $\mathrm{Ayub}^{32}$, que destaca que a posição anterior da cabeça irá acarretar distúrbios de posicionamento e funcionamento mandibular, levando a uma crescente tensão na musculatura mastigatória e conseqüentemente DTM.

Amantea $^{33}$ aborda a importância da avaliação postural em pacientes com alteração na ATM, concluindo que há uma íntima relação entre DTM e alterações na postura corporal, corroborando com os achados do presente estudo. O autor ainda justifica, que pacientes com DTM, podem apresentar também, desvios posturais como anteriorização da cabeça, aumento da lordose cervical e não nivelamento entre os ombros.

Os dados obtidos no presente estudo, entre os grupos, se comparados entre si, não demonstram diferença significante entre os ângulos de cabeça e pescoço, porém, ao realizar a análise individual de cada grupo uma variação foi observada em relação aos valores dos ângulos com a severidade da DTM. Este fato pode ser justificado pelo estudo realizado por Bricot $^{34}$, onde o autor relata que as alterações da ATM estão associadas as desordens crânio-mandibulares e que os dismorfismos craniofaciais apresentam entre seus tipos diferentes classes oclusais, onde a posição da mandíbula tem relação direta com a postura da cabeça e ombros.

O fato do grupo DTM apresentar relação diretamente proporcional, entre o grau de DTM, segundo Fonseca et al. ${ }^{1}$ (leve, moderada e severa) e o aumento do ângulo cervical, esta relação não foi estatisticamente significante, não podendo ser afirmado para esta amostra.

\section{CONCLUSÃO}

Houve uma prevalência maior de Disfunção Temporomandibular (DTM) leve, sendo que em relação ao ângulo cervical, houve um aumento com a severidade do grau de DTM, com piora da qualidade de vida nesta população. 


\section{Agradecimentos}

A Profa. Dra. Luciana Maria Malosá Sam- paio Jorge. Aos alunos Mariana Sampaio; Paula Mendes; Kelly Ikeda; Amanda Sapienza; Thiago Vasconcelos; Marília Bernardi.

\begin{abstract}
Introduction: Temporomandibular dysfunction (TMD) presents several clinical manifestations, with high prevalence of postural changes and impacts on the individual's quality of life. Objective: To characterize the degree of temporomandibular dysfunction (TMD) and to correlate it with cervical posture and the quality of life of undergraduate students. Materials and Methods: Ninety-eight individuals between 18 and 33 years old of both sexes were selected by means of the questionnaires Fonseca's Anamnesis Index, which graduates TMD severity, and the Brazilian version of the SF-36 Quality of Life Questionnaire. Analysis of the head and neck posture was performed with the Alcimagem software, which offers a quantitative analysis of the angles. Results: The data collected demonstrated that $68.36 \%$ of the subjects presented mild TMD $23.47 \%$ presented moderate TMD and $8.16 \%$ presented severe TMD; the participants presented distortions in the Vitality and Pain domains $\left(X^{2}=0.03\right)$, while the domains of Emotional and Social Aspects remained preserved. The average of cervical angle in individuals with TMD was $90.76^{\circ}( \pm 7.72)$, and an increase in cervical angle was observed related to the increase in TMD severity degree; however, this data was not statistically significant $(r=0.09)$. Conclusion: TMD influenced the quality of life of the sample utilized. A possible increase in cervical angle related to an increased TMD severity degree is suggested.
\end{abstract}

Keywords: Temporomandibular joint dysfunction syndrome; quality of life; posture.

\section{REFERÊNCIAS}

1. Fonseca DM, Bonfante G, Valle AL, Freitas SFT. Diagnóstico pela anamnese da disfunção craniomandibular. RGO 1994; 42(1):23-8.

2. Okeson, J. P. Fundamentos de oclusão e desordens temporomandibulares. $2^{\text {a }}$ ed. São Paulo: Artes Médicas, 1992.

3. Nassif NJ, Al-Salleeh F, Al-Admawi M. The prevalence and treatment needs of symptoms and signs of temporomandibular disorders among young adult males. J Oral Rehabil 2003;30:944-950.

4. Boyd Ch, Slagle Wf, Boyd Cm, Bryant Rw, Wiygul Jp. The effect of head position on electromyographic evaluation of representative mandibular positioning muscle groups. J Cranio Practice. 1987;(5)50.

5. Dworkin, SF, LeResche L. Research diagnostic criteria for temporomandibular disorders: review, criteria, examinations and specifications. critique. J Craniomandib Disord 1992;6(4):301-55.

6. Kafas P, Leeson R. Assessment of pain in temporomandibular disorders: the biopsychosocial complexity. Int J Oral Maxillofac Surg 2006; 35(2):145-9.

7. Magnusson T; Egermark I; Carlsson GE. A longitudinal epidemiologic study of signs and symptoms of temporomandibular disorders from 15 to 35 years of age. J Orofac Pain 2000;14(4): 310-9.
8. Lobbezoo F, Drangsholt M, Peck C, Sato H, Kopp $\mathrm{S}$, Svensson P. Topical review: new insights into the pathology and diagnosis of disorders of the temporomandibular joint. J Orofac Pain 2004; 18:181191.

9. John MT, Dworkin SF, Mancl LA. Reliability of clinical temporomandibular disorder diagnoses. Pain 2005;118(1-2):61-9

10. Selaimen CM, Jeronymo JC, Brilhante DP, Grossi ML. Sleep and depression as risk indicators for temporomandibular disorders in a cross-cultural perspective: a case-control study.Int J Prosthodont 2006;19(2):154-61.

11. Oliveira AS, Dias EM, Contato RG, Berzin F. Prevalence study of signs and symptoms of temporomandibular desorders in Brazilian college students. Braz Oral Res 2006; 20(1):3-7.

12. ReisineST, Weber J. The effects of temporomandibular joint disorders on patients' quality of life. Community Dent Health 1989; 6(3):257-270.

13. Bernhardt $\mathrm{O}$ et al.. Risk factors for headache, including TMD signs and symptoms, and their impact on quality of life. Results of the Study of Health in Pomerania (SHIP). Quintessence Int 2005;36(1):55-64.

14. Ciconelli RM, Ferraz BF. Tradução para língua portuguesa e validação do questionário genérico de avaliação de qualidade de vida SF36 (Brasil). 
Rev Bras Reumatol 1999; 39(3):141-150

15. Barauna, M.A; Adorno,M.L. Avaliação Cinesiológica das Curvaturas Lombar e Torácica das Gestantes através do Cigolordômetro e da Fotogrametria Computadorizada e suas Relações com a Dor Lombar. Fsioterapia Brasil,2001, 2(3).

16. Rocabado M. Cabeza e Cuello-Tratamiento Articular. Buenos Aires: Intermédica; 1979.

17. Aaronson NK., Acquadro C., Alonso J., Apolone G., Bucquet D., Bullinger M., Bungay K., Fukuhara S., Gandek B, Keller S., Razavi D., Sanson-Fisher R., Sullivan, M., Wood-Dauphinee S., WagnerA.,. Ware JE. International quality of life assessment (IQOLA) project. Quality of Life Research, 1992 ;1(5)

18. Gadotti, IC, Berzin, F,Biasotto-Gonzalez, Preliminary rapport on head posture and muscle activity in subjects with class I and II. Journal of Oral Rehabilitation 32 (11), 794-799, 2005.

19. Chaves TC, Costa D, Bevilaqua-Grossi D, Bertoli F. Avaliação anamnésica de sintomas de disfunção temporomandibular em crianças asmáticas. Fisioterapia e Pesquisa 2005; 11(1): 19-26.

20. Davidson, M.; Keating, J.L. A Comparison of Five Low Back Disability Questionnaires: Reliability and Responsiveness. Phys Ther, v.82, n.1, p.8-24, 2002.

21. Hays, R. D.; Sherbourner, C. D.; Mazel, R. M. The RAND 36-Item Health Survey 1.0. Health Economics, v.2,p.217-227, 1993.

22. Brazier, J. E.; Harper, R.; Jones, N.M.; O'cathain, A.; Thomas, K.J.; Usherwood, T.; Westlake, L. Validating the SF-36 Health Survey Questionnaire New Outcome Measure for Primary Care. BMJ v.305, p.160-4, 1992.

23. OliveiraAS, Bermudez CC, de Souza RA, Souza CMF, Dias EM, Castro SCE, Bérzin F. Impacto da dor na vida de portadores de disfunção temporomandibular. JAppl Oral Sci, 2003;11(2):138-143.

24. Jedel E, Carlsson J, Stener-Victorin E. Health-related quality of life in child patients with temporomandibular disorder pain. Eur J Pain 2007;11(5):557-563.

25. Dervis E, Dervis E. The prevalence of temporomandibular disorders in patients with psoriasis with or without psoriatic arthritis. J Oral Rehabil 2005;32(11):786-93.

26. Dahlstrom L, Widmark G, Carlsson SG. Cognitivebehavioral profiles among different categories of orofacial pain patients: diagnostic and treatment implications. Eur J Oral Sci. 1997 Oct;105 (5 Pt 1):37783.

27. Kuroiwa DN, Marinelli JG, Nicodemo D, Duarte MSR, Oliveira W.. Indicadores de qualidade de vida relacionados à disfunção temporomandibular e dor orofacia Braz Oral Res 2006;20(Suppl):45.

28. Biasotto-Gonzalez, DA. Abordagem interdisciplinar das disfunções temporomandibulares. São Paulo: Editora Manole, 2005, 246p.

29. Cauás M.,Alves, I.F., Tenório K., Filho JBHC. Incidências de Hábitos Parafuncionais e Posturais em Pacientes Portadores de Disfunção da Articulação Craniomandibular. Revista de Cirurgia e Traumatologia Buco-Maxilo-Facial. 2004 abr/ jun,4(2): $121-129$.

30. Brown FF, Robinson ME, Riley JL, Gremillion HA. Pain severity, negative affect, and microstressers as predictors of life interference in TMD patients. Cranio. 1996 Jan;14(1):63-70.

31. Goldstein DF. et al.. Influense of cervical posture on mandibular movement. The Journal of Prosthetic Dentistry, St. Louis, 1984 Sep.,52(3):421-431.

32. Ayub, E., Glasheen-Wray, M., Krauss, S. Head posture: a case report of the effects on the rest position of the mandible. J Orthoapaedic and Sports Physical Therapy 1984; 5(4):179-60.

33. Amantéa DV., Novaes AP., Campolongo GD., Barros TP. A importância da avaliação postural no paciente com disfunção da articulação temporomandibular. Acta ortop. bras, 2004, jul/set, 12(3):155-159.

34. Bricot B. Posturologia. São Paulo: Ícone; 1999, 270p.

Recebido em: 29/08/2007

Modificado em: 03/11/2007

Aprovado em: 21/02/2008 